Contract No. and Disclaimer:

This manuscript has been authored by Savannah River Nuclear Solutions, LLC under Contract No. DE-AC09-08SR22470 with the U.S. Department of Energy. The United States Government retains and the publisher, by accepting this article for publication, acknowledges that the United States Government retains a non-exclusive, paid-up, irrevocable, worldwide license to publish or reproduce the published form of this work, or allow others to do so, for United States Government purposes. 


\section{Novel Concepts for Isotopic Separation of ${ }^{3} \mathrm{He} /{ }^{4} \mathrm{He}$ Lindsay E. Roy (PI), Holly L. Watson, and H. Lee Nigg}

Abstract: The research outlined below established theoretical proof-of-concept using ab initio calculations that ${ }^{3} \mathrm{He}$ can be separated from ${ }^{4} \mathrm{He}$ by taking advantage of weak van der Waals interactions with other higher molecular weight rare gases such as xenon. To the best of our knowledge, this is the only suggested method that exploits the physical differences of the isotopes using a chemical interaction.

The world is experiencing a shortage of ${ }^{3} \mathrm{He}$, a rare isotope of helium with many different critical applications in medical imaging, cryogenics, oil \& gas exploration, scientific research, and in particular, neutron detection. One potential source for ${ }^{3} \mathrm{He}$ outside of using tritium includes extraction of naturally occurring ${ }^{3} \mathrm{He}$ from natural gas. The U.S. Geological Survey estimates that ${ }^{3} \mathrm{He}$ reserves and resources to be between 1 and 5 million liters. To date, there is no practical method to isolate and recover ${ }^{3} \mathrm{He}$ from ${ }^{4} \mathrm{He}$ on a production scale which, if it existed, could take advantage of untapped resources for this vital isotope. ${ }^{3} \mathrm{He}$ constitutes only $1.37 \mathrm{ppm}$ of total $\mathrm{He}$, too dilute to separate economically from ${ }^{4} \mathrm{He}$ (the major isotope) by distillation alone. 'Superfluid' separations through a filter are feasible at temperatures of $2.17 \mathrm{~K}$ or less. Membrane separation using porous graphene has been suggested but operating temperatures are predicted to be around $10 \mathrm{~K}$. Despite the information taught in general chemistry textbooks, He will form a weak van der Waals interaction with other rare gases (Rg) such as Xe to form a heteronuclear dimer. Using van der Waals interactions as a separations technique could move the process outside of liquid hydrogen temperatures. The research outlined below used a theoretical approach to explore the weak van der Waals interactions of rare gases (such as Xe) with ${ }^{3} \mathrm{He}$ and ${ }^{4} \mathrm{He}$ to determine if the interaction can be used for He isotope separations.

A series of high-level $a b$ initio calculations at different temperatures for the homonuclear rare gas dimer ${ }^{4} \mathrm{He}_{2}$ (benchmarking) and heteronuclear dimers ${ }^{3} \mathrm{He} /{ }^{4} \mathrm{He}-\mathrm{Xe},{ }^{3} \mathrm{He} /{ }^{4} \mathrm{He}-\mathrm{Ar},{ }^{3} \mathrm{He} /{ }^{4} \mathrm{He}-\mathrm{Xe}-$ $\mathrm{Xe},{ }^{3} \mathrm{He} /{ }^{4} \mathrm{He}-\mathrm{Ar}-\mathrm{Ar},{ }^{3} \mathrm{He}_{2} \mathrm{Xe}$, and ${ }^{4} \mathrm{He}_{2}-\mathrm{Xe}$ were performed. $\mathrm{He}_{2}$ is the lightest van der Waals system and is difficult to treat accurately because its dissociation energy is small $(11 \mathrm{~K})$ and the attraction is dominated by dispersion forces. The potential energy curves needed for thermodynamic property prediction were created using highly accurate coupled cluster UCCSD$\mathrm{T}$ correlation energy treatments employing correlation consistent basis sets. This provided excellent compromise between accuracy and computational cost to evaluate the concept as a possible separations technique (calculated ${ }^{4} \mathrm{He}_{2}$ dissociation energy is $10.71 \mathrm{~K}$ ). The thermodynamic predictions were then used to estimate partition function ratios at optimized temperatures. Functions over 1.25 at temperatures above liquid nitrogen $(77 \mathrm{~K})$ may indicate a practical separations process, though a method that takes advantage of this result still needs to be designed.

Figure 1 shows the potential energy curve (top) for a single ${ }^{4} \mathrm{He}$ atom bound to an Ar or Xe atom to create a heterodinuclear molecule. The dissociation energy can be computed from this curve by subtracting the zero point energy from the well depth. The dissociation energy is expressed in wavenumbers and is a measure of the energy required to dissociate the He atom from the rare gas; the larger the number, the stronger the interaction between the atoms. Benchmark calculations show a ${ }^{4} \mathrm{He}-\mathrm{Rg}$ bond dissociation energy of $\sim 22 \mathrm{~cm}^{-1}$ for ${ }^{4} \mathrm{He}-\mathrm{Ar}$ and $\sim 80 \mathrm{~cm}^{-1}$ for ${ }^{4} \mathrm{He}-\mathrm{Xe}$. The higher value for He-Xe indicates a stronger ${ }^{4} \mathrm{He}-\mathrm{Xe}$ attraction than with Ar. The bottom half of Figure 1 shows the formation enthalpy $\left(\Delta \mathrm{H}_{\mathrm{f}}\right)$ as a function of temperature for the 


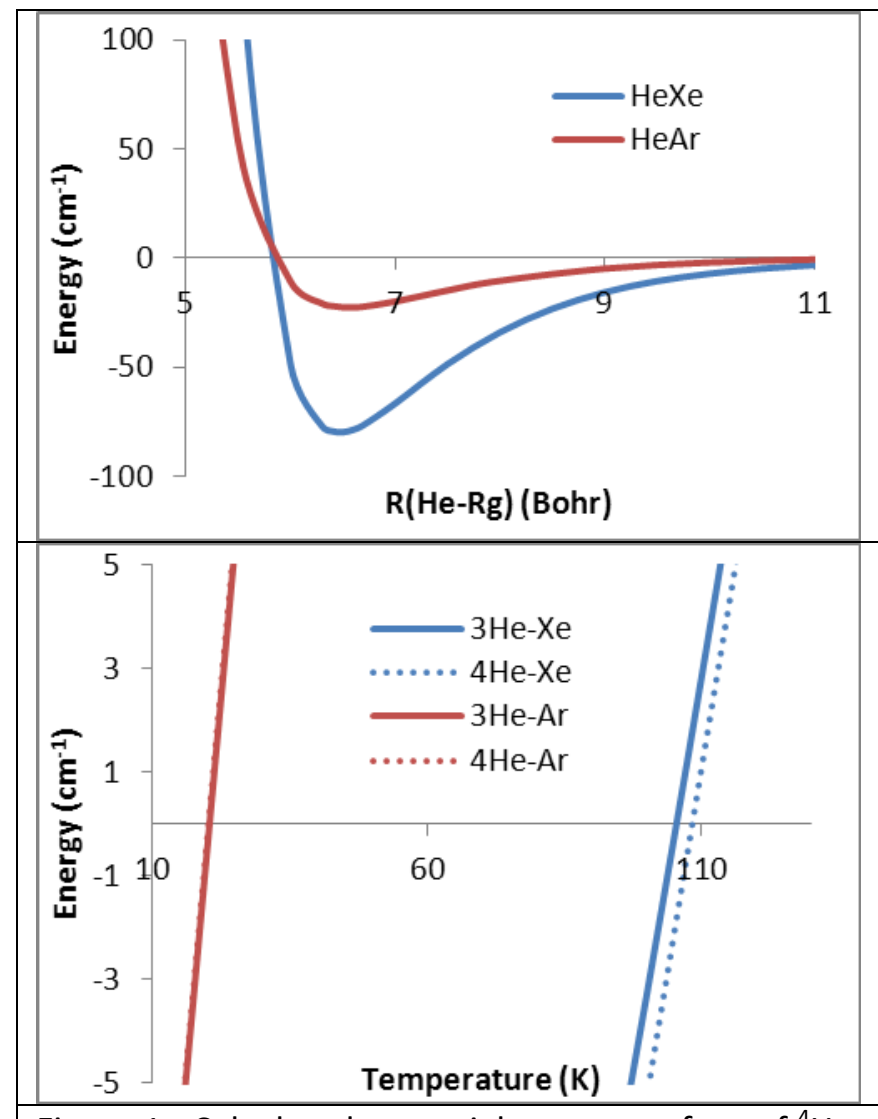

Figure 1. Calculated potential energy surface of ${ }^{4} \mathrm{He}-$ $\operatorname{Rg}(\operatorname{Rg}=\mathrm{Xe}, \mathrm{Ar})$ species as a function of distance (Top) and formation enthalpy $\left(\Delta \mathrm{H}_{\mathrm{f}}\right)$ as a function of temperature (Bottom) for the binding of ${ }^{3} \mathrm{He} /{ }^{4} \mathrm{He}$ with $\mathrm{Ar}$ and Xe at the UCCSD-T level of theory. binding of ${ }^{3} \mathrm{He} /{ }^{4} \mathrm{He}$ with $\mathrm{Ar}$ and $\mathrm{Xe}$. The graph shows that formation of a ${ }^{3} \mathrm{He}-\mathrm{Xe}$ heterodinuclear molecule is predicted to occur at $\sim 105 \mathrm{~K}$ and for ${ }^{4} \mathrm{He}-\mathrm{Xe}$ at $\sim 108 \mathrm{~K}$. In other words, $a b$ initio calculations predict at $3 \mathrm{~K}$ temperature difference between the two species with the ${ }^{4} \mathrm{He}$ isotope having a stronger interaction to $\mathrm{Xe}$. The partition function ratio for ${ }^{4} \mathrm{HeXe} /{ }^{3} \mathrm{HeXe}$ at $100 \mathrm{~K}$ is 1.434. This number is the equilibrium constant for the exchange reaction between the separated atoms and the molecule in question. Separation factors can then be calculated from the partition function ratio for the isotopic molecules involved in a reaction. For comparison, the same calculations were performed for the ${ }^{3} \mathrm{He} /{ }^{4} \mathrm{He}-\mathrm{Ar}$ species, but the temperature difference was negligible. The partition function ratio for ${ }^{4} \mathrm{HeAr} /{ }^{3} \mathrm{HeAr}$ at $20 \mathrm{~K}$ is 1.342 .

These preliminary data are encouraging and suggest that $\mathrm{He}$ isotope separations could occur using van der Waals interactions with Xe. Xe becomes a solid at $161 \mathrm{~K}$ under atmospheric pressure (above the heterodinuclear formation temperature).

Further investigation is needed to determine if ideal separation system working conditions would be in the liquid, gas, or supercritical phase. Follow-on funding will be requested to perform additional calculations to improve calculation fidelity, estimate temperature/pressure requirements and to design a benchtop separation system. The benchtop separation system is necessary to demonstrate proof-of-concept of the $a b$ initio results. This may require refined calculation to determine the appropriate operating conditions for the system. Ideally, the system would include a mechanism to separate and recycle Xe. The separation products would be verified using IR and mass spectrometry. 